\title{
Conhecendo as Crenças sobre Inteligência, Esforço e Sorte de Alunos Brasileiros em Tarefas Escolares
}

\author{
Evely Boruchovitch 123 \\ Universidade Estadual de Campinas \\ Universidade São Francisco
}

\begin{abstract}
Resumo
Este trabalho teve como objetivo investigar crenças sobre inteligência, esforço e sorte entre escolares do ensino fundamental. A amostra foi composta de 110 alunos de terceira, quinta e sétima séries de uma escola pública de Campinas. Os sujeitos eram de ambos os sexos e provenientes de famílias de nível sócio-econônico desfavorecido. Os sujeitos foram entrevistados individualmente e seus conceitos foram mensurados por questões abertas e fechadas. A inteligência foi concebida predominantemente como sabedoria. O esforço foi definido em termos de comportamentos relativos ao estudar. Já a sorte foi associada a ganhar ou achar coisas inesperadamente. Relações significativas entre as variáveis investigadas, idade, série escolar, gênero e repetência escolar dos alunos foram encontradas. Discute-se a importância de se conhecer e se compreender mais sistematicamente o desenvolvimento de crenças como inteligência, esforço e sorte em crianças brasileiras, dada a relevância dos mesmos para a psicologia escolar e a carência de estudos brasileiros nessa área.
\end{abstract}

Palavras-chave: Crenças; inteligência; esforço; sorte; Ensino Fundamental.

Brazilian Students' Beliefs about Intelligence, Effort and Luck in School Tasks

\begin{abstract}
The objective of this paper is to investigate the beliefs of intelligence, effort and luck among Brazilian students. The sample was composed of 110 students of third, fifth, and seventh grade of a public school of Campinas. Subjects were from both sexes and from low socio-economic background. Participants were interviewed individually and their beliefs were measured by opened and closed-end questions. Intelligence was predominantly conceptualized as wisdom. Effort was associated with behaviors related to studying and luck was defined as winning or finding something unexpectedly. Significant relationships were found among the beliefs investigated and age, school grade level, gender and repetition of a school grade level. The importance of knowing and comphreending the development of beliefs about intelligence, effort and luck in Brazilian students is discussed, given the relevance of such beliefs for educational psychology, as well as the lack of such studies in Brazil.

Keywords: Beliefs; intelligence; effort; luck; elementary school.
\end{abstract}

As teorias cognitivas da motivação para a realização assumem que um comportamento é determinado pelas crenças individuais do aluno. Essas crenças funcionam como mediadoras do comportamento (Bandura, 1982, 1989), sejam elas baseadas numa realidade objetiva ou subjetiva (Givvin, Stipek, Salmon \& MacGyvers, 1998).

A inteligência, o esforço, a sorte e a dificuldade da tarefa são, segundo Weiner $(1979,1985)$ os fatores mais utilizados pelas pessoas para explicar as causas de suas experiências de sucesso e de fracasso em tarefas acadêmicas. De acordo com a teoria da atribuição de

\footnotetext{
${ }^{1}$ Endereço para correspondência: Rua Coronel Quirino, 910/24, Cambuí, 13.025-001, Campinas, SP. E-mail: evely@obelix.unicamp.br

2 Parcialmente apresentado no V Congresso Nacional de Psicologia Escolar e Educacional, Universidade do Vale do Itajaí, Santa Catarina, abril de 2000. Apoio financeiro CNPq (Proc. No 300162/95-2) e FAEP/Unicamp (Proc. No 0099/00).

3 A autora agradece as valiosas críticas e sugestões de revisores anônimos dessa revista.
}

causalidade (Weiner, 1979, 1985, 1993), as causas são entendidas como tendo três dimensões: localização, estabilidade e controlabilidade. Em termos da localização, uma causa pode ser considerada como externa ou interna (fatores que se encontram fora ou dentro do indivíduo). No que concerne a controlabilidade, uma causa pode ser vista como estando dentro ou fora do controle da pessoa. No que diz respeito a estabilidade, uma causa pode ser entendida como sendo permanente ou passível de mudança. De modo geral, pesquisas revelam que a inteligência/capacidade é vista como uma causa interna (pertencente ao indivíduo), estável (imutável) e fora do controle do mesmo. O esforço é concebido como uma causa interna, instável (pode ser alterado) e controlável (pode ser monitorado) pelo participante. Tanto a sorte quanto a dificuldade da tarefa são vistas como externas, instáveis e incontroláveis (Weiner, 1985).

Como descrito em Boruchovitch (2001), Dweck e Bempechat (1983) defendem a idéia de que o indivíduo 
desenvolve teorias pessoais acerca da sua própria inteligência. Para Dweck e Bempechat (1983), há dois tipos de concepções pessoais sobre a inteligência: a inteligência tida como uma entidade e a visão incremental da inteligência. Enquanto que na teoria da inteligência como uma entidade, o indivíduo acredita que a inteligência é um traço imutável e fixo, os adeptos da visão incremental compartilham a crença de que a inteligência pode ser desenvolvida e ampliada mediante o esforço e a prática. Não há dúvidas de que estas teorias, por sua vez, influenciam a motivação, o comportamento e as ações das pessoas.

Evidências sugerem que crianças bem jovens, como as pré-escolares, sejam capazes de atribuir causas aos eventos que lhes acontecem (Piccinini, 1989), bem como refletir sobre eventos que são controláveis e não controláveis (Kopp, 1982). Estudos realizados com adultos indicam que estes percebem conceitos como inteligência, esforço, entre outros, como distintos, (Nicholls \& Miller, 1984) ao passo que essas idéias parecem um pouco confusas e correlacionadas em crianças (Blumenfield, Pintrich \& Hamilton, 1986).

Constata-se ainda que as crianças mais novas tendem a superestimar suas capacidades, enquanto que crianças mais velhas são mais inclinadas a fazer auto-julgamentos de capacidade mais realistas. De modo geral, as crianças de séries mais elementares não expressam a idéia de que o esforço leva a mudanças na inteligência (Harari \& Covington, 1981), tendem a elaborar julgamentos sobre a própria capacidade baseando-se em pistas de esforço e a considerar a capacidade como instável (Little, 1985). Alunos mais velhos acreditam que a inteligência interage com o esforço para produzir os resultados e concebem a inteligência como mais estável, ao passo que o esforço como mais variável (Rosenholtz \& Simpson, 1984). Stipek e Daniels (1988) mencionam que as crianças mais jovens são mais otimistas sobre suas competências quando comparadas com as mais velhas. Além disso, diferentemente dos adultos que atribuem seus sucessos a causas internas e fracassos a fatores externos (Kelley \& Michella,1980), crianças são, de modo geral, propensas a atribuir mais os seus fracassos do que os seus sucessos a causas internas (Bar-Tal \& Darom, 1979; Frieze \& Snyder, 1980).

Tendo em vista que a motivação para aprendizagem é altamente influenciada pelas crenças individuais dos alunos, bem como que a inteligência é constructo central em nossa cultura, entender como crianças definem conceitos chaves como inteligência, esforço e sorte se constitui num tópico importante não só para a pesquisa e prática educacional, mas também para a psicologia do desenvolvimento. Não há dúvidas, entretanto, que essa temática tem sido pouco explorada, sobretudo na realidade brasileira (Boruchovitch, 1995; Boruchovitch, 1998; Boruchovitch \& Martini, 1997; Martini \& Boruchovitch, 1999; Neves \& Almeida, 1996; Nunes, 1990; Taliuli, 1982). Em conseqüência, o objetivo do presente trabalho é investigar as crenças de escolares brasileiros sobre inteligência, esforço e sorte nas tarefas acadêmicas, bem como avaliar a existência de diferenças nessas crenças, com relação à idade, ao gênero, à série e à repetência escolar do grupo pesquisado.

\section{Método}

\section{Seleção da Escola}

Inicialmente, foi realizado um contato com a Prefeitura de Campinas com o propósito de se obter informações sobre as escolas públicas que mais enfrentam problemas sérios, como repetência e evasão escolar. Uma escola, apresentando os problemas anteriormente mencionados, foi selecionada de uma lista contendo o nome de todas as escolas públicas da região. Sugestões provenientes da chefe da divisão de pesquisa e planejamento da Prefeitura foram também levadas em conta na seleção da escola. A escola escolhida atendia a uma população de baixa renda. A diretora dessa escola foi contactada e os objetivos da pesquisa foram explicitados, bem como o interesse da escola em participar do estudo foi confirmado. A diretora foi informada do carácter confidencial da pesquisa e assegurada de que a coleta de dados transcorreria de acordo com os horários de maior conveniência da escola, de modo a interferir minimamente com a rotina da mesma. Depois que a escola concordou em participar da pesquisa a lista de todos os alunos de terceira, quinta e sétima série foi obtida.

\section{Participantes}

A amostra foi composta de 110 alunos de terceira série $(n=36)$, quinta $(n=38)$ e sétima $(n=36)$ séries do ensino fundamental de uma escola municipal de Campinas. Os participantes eram de ambos os sexos e provenientes de famílias de nível sócio-econômico desfavorecido. A faixa etária dos participantes variou de oito a 16 anos. Mais precisamente, $25,5 \%$ da amostra encontrava-se entre oito e dez anos (grupo 1), 45,5\% entre 11 e 13 anos (grupo 2) e $29,1 \%$ possuía 14 ou mais anos de idade (grupo 3). Em relação à história escolar, 68,1\% dos participantes já haviam sido retidos em pelo menos uma série escolar.

\section{Procedimentos}

\section{Coleta de Dados}

Os participantes foram selecionados aleatoriamente, por sorteio, de 19 classes de terceira, quinta e sétima 
série da escola. Uma divisão balanceada por gênero foi também obtida. Dados sobre a história escolar dos alunos (promoções e retenções) foram conseguidos através dos arquivos da escola e no contato com os alunos. Os participantes foram entrevistados individualmente pela autora e suas crenças foram medidas por questões abertas e fechadas, como as que se seguem: a) O que é inteligência?; b) Você acha que inteligência é importante para alguém tirar boas notas na escola? ( ) sim ( ) não; c) Você acha que inteligência é uma coisa que nós já nascemos com ou é alguma coisa que nós conseguimos depois na vida, na escola? (teoria sobre a inteligência); d) Você acha que uma pessoa que é inteligente é sempre inteligente ou depende da situação? (estabilidade); e) Você acha que inteligência é uma coisa que está dentro ou fora de nós? (locus); f)Você acha que tem alguma coisa que uma pessoa possa fazer para ser uma pessoa inteligente? ( ) sim ( )não (controlabilidade)? Questões semelhantes foram utilizadas para medir as crenças relativas ao esforço e a sorte. As repostas dos alunos foram transcritas na íntegra.

O procedimento de coleta de dados e as questões utilizadas no presente estudo foram revisados e refinados, levando-se em conta as informações provenientes de um estudo piloto previamente realizado com 12 participantes (quatro de cada série) da mesma escola que não fizeram parte da amostra. A autora estabeleceu um rapport com os participantes. Os participantes foram informados que eles foram sorteados para participar de uma pesquisa voltada para compreender melhor o que eles pensam a respeito de questões relacionadas à própria aprendizagem. Os participantes foram assegurados do carácter confidencial do estudo e de que as respostas fornecidas não iriam influenciar nas suas notas, já que os dados coletados só seriam utilizados para a pesquisa. Os alunos eram livres para participar ou não. $\mathrm{O}$ desinteresse por fazer parte do estudo foi raro. Nesse caso, sorteava-se um outro estudante.

\section{Resultados}

As respostas das questões abertas foram estudadas por análise de contéudo. No que concerne às questões abertas, cada aluno pode dar mais de uma resposta. Sistemas de categorização das respostas foram desenvolvidos. Uma amostra das respostas de cada uma das questões abertas foi categorizada também por um juiz independente. A consistência entre a pesquisadora e o juiz variou de 80 a 100\%. Primeiramente, a frequência e a porcentagem de respostas em cada categoria, foram calculadas. Em um segundo momento, procedimentos da estatística inferencial foram utilizados para analisar as relações entre as crenças dos estudantes sobre inteligência, esforço e sorte e, a idade, a série escolar, o gênero, e a repetência escolar do grupo pesquisado. Dada a natureza categórica das variáveis investigadas, a prova do QuiQuadrado foi realizada.

\section{Crenças sobre a Inteligência}

A inteligência foi definida pelos participantes predominantemente como Sabedoria $(47,1 \%)$. A concepção de inteligência como capacidade para aprender $(15,2 \%)$ e a idéia de inteligência associada a Comportamentos relativos ao Estudo (14,4\%) foram

Tabela 1. Porcentagem das Respostas dos Alunos para a Questão "O que é Inteligência?”

\begin{tabular}{llc}
\hline Categorias & $n$ & $\%$ \\
\hline Capacidade & 21 & 15,2 \\
Estudo & 20 & 14,5 \\
Juízo de Valor & 19 & 13,7 \\
Não Sabe & 13 & 9,4 \\
Sabedoria & 65 & 47,1 \\
Total & 138 & 100 \\
\hline
\end{tabular}

Tabela 2. Porcentagem de Respostas para a Questão “O que é Inteligência?” por Idade

\begin{tabular}{lccccc}
\hline Idade & Sabedoria & Capacidade & $\begin{array}{c}\text { Inteligência } \\
\text { Estudo }\end{array}$ & Juízo & Não Sabe \\
\hline $8-10$ & 18,8 & 9,5 & $40,0 *$ & 26,3 & 23,1 \\
$11-13$ & 51,6 & 57,1 & 55,0 & 57,9 & 38,5 \\
$>14$ & 29,7 & 33,3 & 5,0 & 15,8 & 38,5 \\
Total & 100,0 & 100,0 & 100,0 & 100,0 & 100,0 \\
\hline
\end{tabular}

$N=110$

$* p<0,05$

$* * p<0,01$ 
encontradas apenas numa minoria das respostas (Tabela 1). A prova do Qui-Quadrado revelou relações significativas entre a categoria Comportamentos relativos ao Estudo e a idade dos participantes, $\chi 2=8,33 ; p=0,016$. Como pode ser visto na Tabela 2 , a porcentagem de respostas nesta categoria foi mais alta entre os alunos mais jovens, quando comparados aos alunos mais velhos.

\section{Crenças sobre o Esforço}

Os dados na Tabela 3 revelam que o esforço foi definido pelos participantes predominantemente em termos de Comportamentos relativos ao Estudo (32,5\%) e em termos de Luta/Persistência para Conseguir as Coisas (30,8\%). Esforço relacionado à idéia de Vontade/ Querer Aprender ocorreu também em menor frequência (17,5\%). Relações significativas entre a categoria Luta/Persistência, idade, $\chi 2=5,98$; $p=0,04$, e série escolar, $\chi 2=7,87 ; p=0,019$, foram encontradas. O percentual de respostas nesta categoria foi mais alto entre os alunos mais velhos e mais avançados na escolaridade (Tabela 4).

Tabela 3. Porcentagem das Respostas dos Alunos para a Questão "O que é Esforço?”

\begin{tabular}{llc}
\hline Categorias & $n$ & $\%$ \\
\hline Estudo & 39 & 32,5 \\
Lutar & 37 & 30,8 \\
Não Sabe & 16 & 13,3 \\
Comptos Sociais & 07 & 5,8 \\
Vontade & 29 & 17,5 \\
Total & 120 & 100 \\
\hline
\end{tabular}

\section{Crenças sobre a Sorte}

A Tabela 5 demonstra que a sorte foi conceituada como Achar ou Ganhar Alguma Coisa Inesperadamente pela maioria dos participantes $(42,6 \%)$. Respostas em que a Sorte foi definida em termos de Juízo de Valor (isto é, respostas que associaram a sorte a ter uma família ou que emitiram um julgamento em relação a sorte do tipo: é bom; não acredito em sorte) também foram encontradas $(22,1 \%)$. O percentual de respostas na categoria Não Sabe foi considerável $(21,6 \%)$. Nenhuma relação significativa emergiu entre as categorias de respostas e as variáveis demográficas.

Tabela 5. Porcentagem das Respostas dos Alunos para a Questão "O que é Sorte?”

\begin{tabular}{lcc}
\hline Categorias & $n$ & $\%$ \\
\hline Achar/Ganhar & 52 & 42,6 \\
Estudo & 17 & 13,9 \\
Não Sabe & 26 & 21,7 \\
Juízo de Valor & 27 & 22,1 \\
Total & 122 & 100 \\
\hline
\end{tabular}

\begin{abstract}
A Importância, a Teoria Pessoal, o Locus, a Controlabilidade e a Estabilidade das Crenças sobre Inteligência, Esforço e Sorte

Os dados da Tabela 6 indicam que a inteligência, o esforço e a sorte foram considerados pelos participantes como fundamentais para o sucesso na escola. Em termos do locus da causalidade, os conceitos de inteligência, esforço e sorte foram vistos pela amostra como predominantemente internos. A inteligência, o esforço e a sorte foram considerados como instáveis e dependentes da situação pela maioria dos participantes. A maioria dos participantes
\end{abstract}

Tabela 4. Porcentagem de Respostas dos Alunos para a Questão “O que é Esforço?” por Idade e Série Escolar

\begin{tabular}{ccccccc}
\hline & & & & Esforço & & \\
& & Lutar & Estudar & Vontade & Juízo/Social & Não Sabe \\
\hline \multirow{3}{*}{ Idade } & $8-10$ & $10,8^{*}$ & 23,6 & 23,8 & 0 & 43,8 \\
& $11-13$ & 48,6 & 44,7 & 42,9 & 71,4 & 31,3 \\
\multirow{2}{*}{ Total } & $>14$ & 40,5 & 28,9 & 33,3 & 28,6 & 25,0 \\
& & 100 & 100 & 100 & 100 & 100 \\
Série & $3 \mathrm{a}$ & $16,2 *$ & 43,6 & 33,3 & 42,9 & 37,5 \\
& $5 \mathrm{a}$ & 40,5 & 35,9 & 19,0 & 14,3 & 43,8 \\
Total & $7 \mathrm{a}$ & 43,2 & 20,5 & 47,6 & 42,9 & 18,8 \\
\hline
\end{tabular}

$N=110$

$* p<0,05$

$* * p<0,01$ 
acredita que há algo que podem fazer para serem pessoas inteligentes, esforçadas e sortudas (Tabela 6).

Tabela 6. Crenças sobre Inteligência, Esforço e Sorte da Amostra Total quanto à Importância, à Teoria Pessoal, ao Locus, à Controlabilidade e à Estabilidade

\begin{tabular}{llrrr}
\hline & & \multicolumn{3}{c}{ Conceitos } \\
& & Inteligência & Esforço & Sorte \\
\hline \multirow{3}{*}{ Importância } & Sim & 90,8 & 96,4 & 60,0 \\
& Não & 9,2 & 3,6 & 40,0 \\
& Total & 100,0 & 100,0 & 100,0 \\
\multirow{4}{*}{ Teoria } & Nasce & 24,5 & 13,6 & 43,6 \\
& Consegue & 75,5 & 86,4 & 56,4 \\
& Total & 100,0 & 100,0 & 100,0 \\
Locus & Dentro & 89,1 & 80,9 & 64,5 \\
& Fora & 10,9 & 19,1 & 35,5 \\
\multirow{4}{*}{ Controlabilidade } & Não & 18,2 & 20,0 & 66,4 \\
& Total & 100,0 & 100,0 & 100,0 \\
\multirow{4}{*}{ Estabilidade } & 81,8 & 80,0 & 33,6 \\
& Total & 100,0 & 100,0 & 100,0 \\
& Sempre & 28,2 & 43,6 & 20,0 \\
& Depende & 71,8 & 56,4 & 80,0 \\
& Total & 100,0 & 100,0 & 100,0 \\
\hline
\end{tabular}

A Importância, a Teoria Pessoal, o Locus, a Controlabilidade e a Estabilidade das Crenças sobre Inteligência, Esforço e Sorte em Relação à Idade, à Série Escolar, à Repetência e ao Gênero

Relações significativas foram encontradas entre a importância da inteligência para o sucesso escolar e a série escolar dos participantes, $\chi 2=7,54 ; p=0,023$. Alunos de terceira e quinta série consideraram mais a inteligência como importante para o sucesso escolar, respectivamente, do que alunos de $7 \mathrm{a}$ série. A importância da sorte para o sucesso escolar também encontrou-se significativamente associada a idade, $\chi 2=9,57 ; p=0,008$, e à série escolar, $\chi 2=14,90 ; p=0,001$, dos participantes. Mais precisamente, a importância da sorte para o sucesso escolar dimimui com o aumento da idade e com o avançar da escolaridade (Tabela 7). Como pode ser visto na Tabela 8, a concepção de inteligência, vista como algo que já se nasce com, foi significativamente mais alta entre os alunos mais velhos, $\chi 2=6,89 ; p=0,032$. Para os repetentes, a inteligência foi concebida significativamente mais, $\chi 2=4,49 ; p=0,029$, instável do que para os não repetentes. Encontrou-se ainda, uma relação significativa entre a controlabilidade da inteligência e o gênero, $\chi 2=5,06 ; p=0,022$. Quando comparados com o sexo masculino, os participantes do sexo feminino consideraram a inteligência muito mais como algo que se tem controle (Tabela 8).

Tabela 7. Crenças sobre a Importância da Inteligência, do Esforço e da Sorte para o Sucesso Escolar em relação à Idade e à Série Escolar

\begin{tabular}{llcrc}
\hline & & \multicolumn{3}{c}{ Importância } \\
& & Inteligência & Esforço & \multicolumn{1}{c}{ Sorte } \\
\hline \multirow{4}{*}{ Idade } & $8-10$ & 24,2 & 23,8 & $31,8^{* *}$ \\
& $11-13$ & 49,5 & 47,6 & 47,0 \\
& $>=14$ & 26,3 & 28,6 & 21,2 \\
& Total & 100,0 & 100,0 & 100,0 \\
& $3 \mathrm{a}$ & $36,0 *$ & 34,9 & $47,0 * *$ \\
Série & $5 \mathrm{a}$ & 36,0 & 34,9 & 31,8 \\
& $7 \mathrm{a}$ & 28,0 & 30,2 & 21,2 \\
& Total & 100,0 & 100,0 & 100,0 \\
\hline
\end{tabular}

Tabela 8. Porcentagem das Respostas sobre a Estabilidade e Controlabilidade e Teoria Pessoal da Inteligência em Relação à Repetência, ao Gênero e à Idade

\begin{tabular}{|c|c|c|c|c|c|c|c|}
\hline & & $\begin{array}{c}\text { Estabilidade } \\
\text { Sempre }\end{array}$ & $\begin{array}{l}\text { Inteligência } \\
\text { Depende }\end{array}$ & $\begin{array}{c}\text { Controlabilidade } \\
\text { Não }\end{array}$ & Sim & $\begin{array}{l}\text { Teoria } \\
\text { Nasce }\end{array}$ & $\begin{array}{c}\text { Pessoal } \\
\text { Consegue }\end{array}$ \\
\hline & Sim & 45,2 & $67,1^{*}$ & 70,0 & 58,1 & 59,3 & 61,4 \\
\hline \multirow[t]{3}{*}{ Repetência } & Não & 54,8 & 32,9 & 30,0 & 41,1 & 40,7 & 38,6 \\
\hline & Total & 100,0 & 100,0 & 100,0 & 100,0 & 100,0 & 100,0 \\
\hline & Masculino & 45,2 & 54,8 & 70,0 & $30,0 *$ & 37,0 & 50,6 \\
\hline \multirow[t]{3}{*}{ Gênero } & Feminino & 48,1 & 51,9 & 42,2 & 57,8 & 63,0 & 49,4 \\
\hline & Total & 100,0 & 100,0 & 100,0 & 100,0 & 100,0 & 100,0 \\
\hline & $8-10$ & 19,4 & 24,4 & 20,0 & 22,9 & $11,1 *$ & 26,8 \\
\hline \multirow[t]{3}{*}{ Idade } & $11-13$ & 45,2 & 48,7 & 55,0 & 47,7 & 40,7 & 50,0 \\
\hline & $>=14$ & 35,5 & 26,9 & 25,0 & 29,4 & 48,1 & 23,2 \\
\hline & Total & 100,0 & 100,0 & 100,0 & 100,0 & 100,0 & 100,0 \\
\hline
\end{tabular}

$N=110$

$* p<0,05$

$* * p<0,01$ 
Os dados da Tabela 9 revelam que o esforço foi visto significativamente, $\chi 2=8,55 ; p=0,004$, mais como algo que nós conseguinos depois na vida pelos repetentes do que pelos não repetentes. A sorte foi significativamente, $\chi^{2}=4,59 ; p=0,025$, mais vista como interna pelos não repetentes do que pelos repetentes (Tabela 10).

Tabela 9. Porcentagem das respostas relativas à Teoria Pessoal sobre o Esforço em relação à Repetência

\begin{tabular}{lllc}
\hline & & \multicolumn{2}{c}{ Teoria } \\
& & Nasce & Consegue \\
\hline Repetência & Sim & 26,7 & 66,3 \\
& Não & 73,3 & 33,7 \\
\hline
\end{tabular}

Tabela 10. Porcentagem das Resposta sobre Locus da Sorte em Relação à Repetência

\begin{tabular}{llll}
\hline & & \multicolumn{2}{c}{ Locus } \\
& & Interno & Externo \\
\hline Repetência & Sim & 53,5 & 74,4 \\
& Não & 46,5 & 25,6 \\
\hline
\end{tabular}

\section{Conclusão}

De modo geral, o estudo demonstra que a inteligência é considerada predominantemente como sabedoria, o esforço é definido, tanto em termos de comportamentos ligados ao Estudo, quanto em termos da idéia de Luta/ Persitência e a sorte é concebida como achar ou ganhar alguma coisa inesperadamente. Ao contrário do que seria esperado pela literatura (Blumenfield, Pintrich \& Hamilton, 1986), esses três conceitos revelam-se bastante diferenciados entre os participantes e são definidos em função de critérios bem distintos. Enquanto que a idade, a série escolar e o fato do participante ser ou não repetente mostraram-se bons preditores de diferenças nas crenças da amostra, o gênero exerceu muito pouco impacto.

Embora os participantes tenham sido unânimes em considerar o esforço como um importante determinante do sucesso escolar, os dados levantam questões acerca do porquê a importância da inteligência decai com a idade e a série escolar. Isso possivelmente se deve ao fato de que, diferentemente das crianças novas que não expressam a idéia de que o esforço leva a mudanças na inteligência, crianças mais velhas julgam que a inteligência age em conjunto com o esforço para produzir os resultados. A inteligência foi considerada como instável pela maioria dos participantes, no presente estudo. Todavia, tal como na investigação de Rosenholtz e Simpson (1984), uma concepção de inteligência mais estável com a idade também foi encontrada.

Contrário às expectativas, a importância da sorte foi superestimada entre os participantes, embora esse resultado tenha decaído com a idade, o que seria de se esperar. Isso pode estar relacionado ao próprio desenvolvimento cognitivo dos participantes. Evidências sugerem que as crianças realizam julgamentos mais apropriados com o avançar da idade e da escolaridade (Blumenfield, Pintrich \& Hamilton, 1986), o que é também confirmado pelos resultados do presente estudo, já que o esforço é mais definido em termos de luta/persistência e a inteligência menos em termos unicamente de comportamentos associados ao estudo por participantes mais velhos.

Quanto às três dimensões (locus, estabilidade e controlabilidade), os conceitos investigados no presente estudo foram vistos pela maioria dos participantes de modo diferente do proposto pela Teoria de Atribuição de Causalidade (Weiner, 1985), o que reforça ainda mais a importância de que futuras pesquisas sejam conduzidas no sentido de se conhecer de modo mais aprofundado essas crenças em crianças, ao invés de se utilizar resultados provenientes de pesquisas com adultos (Piccinini, 1989, 1990).

O fato da maioria dos participantes possuir a crença de que eles podem fazer algo para serem pessoas inteligentes e esforçadas abre, indubitavelmente, importantes perspectivas educacionais. A importância de que professores e educadores discutam e trabalhem mais conceitos como inteligência, esforço e sorte dentro da escola torna-se evidente (Boruchovitch \& Martini, 1997). É sabido que o contexto educacional exerce um papel fundamental no desenvolvimento de crenças compatíveis ou não a uma motivação para aprendizagem adequada. Como sugerem Stipek e Daniels (1988), futuros estudos devem ser voltados para o exame do impacto de variáveis pessoais, em conjunto com as variáveis contextuais, nas crenças dos alunos.

Evidências sugerem que as teorias implícitas de professores sobre a natureza da inteligência influenciam sobremaneira a prática pedagógica dos mesmos (Dweck \& Bempechat, 1983; Lynott \& Woolfolk, 1994). Na realidade, os professores têm pouco conhecimento de como a inteligência humana se expressa, bem como desconhecem o real papel do contexto educacional no desenvolvimento da mesma (Mettrau \& Matias, 1998). Professores também utilizam o esforço como se fosse uma palavra mágica e transmitem aos alunos mensagens ambivalentes quanto ao alcance do mesmo, desconhecendo suas limitações (Boruchovitch, 1994). Faz-se necessário que pesquisas sejam direcionadas a investigar mais detalhadamente as crenças 
dos professores acerca da contribuição da inteligência e do esforço para o sucesso escolar.

Como aponta Rosenholtz e Simpson (1984), a formação do conceito de inteligência não depende só do desenvolvimento cognitivo, mas também tem que ser vista como uma construção social. Isso remete-nos a uma reflexão não só acerca de como podemos criar condições que permitam aos alunos aprender a interpretar suas capacidades e limitações de modo mais realístico, mas também sobre como ajudá-los a ampliar a consciência de que a inteligência não é fixa, nem estável e sim, altamente passível de ser desenvolvida pela qualidade das intervenções educacionais (Almeida, 1992).

\section{Referências}

Almeida, L. S. (1992). Inteligência e aprendizagem: Dos seus relacionamentos à sua promoção. Psicologia: Teoria e Pesquisa, 8, 277-292.

Bandura, A. (1982). Self-efficacy mechanism in human agency. American Psychologist, 37, 122-147.

Bandura, A. (1989). Regulation of cognitive processes through perceived self-efficacy. Development Psychology, 25, 729-735.

Bar-Tal, D. \& Darom, E, (1979). Pupils attribution of success and failure. Child Development, 50, 264-267.

Blumenfeld, P. C., Pintrinch, P. R. \& Hamilton, V. L. (1986). Children's concepts of ability, effort and conduct. American Educational Research Journal, 28, 95-104.

Boruchovitch, E. (1994). As variáveis psicológicas e o processo de aprendizagem: Uma contribuição para a psicologia escolar. Psicologia: Teoria e Pesquisa, 10, 129-139.

Boruchovitch, E. (1995). A identificacãa e o estudo das variáveis associadas ao fracasso escolar brasileiro. Projeto de pesquisa (CNPq -Processo No. 300162/95-2). Faculdade de Educação, Departamento de Psicologia Educacional, Universidade Estadual de Campinas. Campinas, S.P.

Boruchovitch, E. (1998, Agosto). Causal attributions for success and failure in Mathematics: A cross-cultural analyses. Trabalho apresentado no 24th International Congress of Applied Psychology, São Francisco.

Boruchovitch, E. (2001). Inteligência e motivação: Perspectivas atuais. Em E. Boruchovitch \& J. A. Bzuneck (Orgs.), Motivação do aluno: Contribuições da psicologia contemporânea (pp. 96-115). Petrópolis: Editora Vozes.

Boruchovitch, E. \& Martini, M. L. (1997). As atribuições de causalidade para o sucesso e o fracasso escolar e a motivação para a aprendizagem de crianças brasileiras. Arquivos Brasileiros de Psicologia, 49(3), 59-71.

Dweck, C. \& Bempechat, J. (1983). Children's theories of intelligence: Consequences for learning. Em S. Paris, G. Olson \& H. Stevenson (Orgs.), Learning and motivation in the classroom (pp. 239-255). Hillsdale, NJ: Erlbaum.

Frieze, I. H. \& Snyder, H. N. (1980). Children's beliefs about the causal of success and failure in school setting. Journal of Educational Psychology, 72, 186-196.
Givvin, K. B., Stipek, D. J., Salmon, J. M. \& Mac Gyvers, V. L. (1998). In the eyes of the bebolder: Students' and teacher's judgments of students' motivation. Manuscrito não publicado. University of Southern Califórnia (UCLA), Los Angeles, Califórnia, EUA.

Harari, O. \& Covington, M. V. (1981). Reactions to achievement behavior from teacher and students perspective: A developmental analysis. American Educational Research Journal, 18, 15-28.

Kelley, H. \& Michella, J. L. (1980). Attribution theory and research. Em M. R. Rosenzweig \& L. M. Porter (Orgs.), Annual Review of Psychology (Vol.31, pp. 457-501). Palo Alto, Annual Review.

Kopp, C. B. (1982). Antecedents of self-regulation: A developmental perspective. Developmental Psychology, 18, 199-214.

Little, A. W. (1985). The child's understanding of the causes of success and failure: A case study of British school children. British Journal of Educational Psychology, 55, 11-23.

Lynott, D. J. \& Woolfolk, A. E. (1994). Teachers' implicit theories of intelligence and their educational goals. The Journal of Research and Development in Education, 27, 253-264.

Martini, M. L. \& Boruchovitch, E. (1999). As atribuições de causalidade, odesenvolvimento infantil e o contexto escolar. Psico-USF, 4 (2), 2336.

Mettrau, M. B. \& Mathias, M. T. (1998). O papel social da prática pedagógica do professor na promoção das capacidades sócio-cognitivo-afetivas do alunado. Tecnologia Educacional, 26(141), 30-34.

Neves, M. B. J. \& Almeida, S. F. C. (1996). O fracasso escolar na 5a série, na perspectiva de alunos repetentes, seus pais e professores. Psicologia: Teoria e Pesquisa, 12, 147-156.

Nicholls, J. G. \& Miller, A. T. (1984). Reasoning about the ability of self and others: A developmental study. Child Development, 55, 1900-1999.

Nunes, A. N. de A. (1990). Fracasso escolar e desamparo adquirido. Psicologia: Teoria e Pesquisa, 6, 139-154.

Piccinini, C. A.(1990). Problemas metodológicos nas investigações sobre atribuições de causalidade para sucesso e fracasso em crianças. Arquivos Brasileiros de Psicologia, 42, 23-29.

Piccinini, C. A. (1989). Atribuições de causalidade em crianças: A alguns aspectos críticos. Psicologia: Teoria e Pesquisa, 5, 57-69.

Rosenholtz, S. J. \& Simpson, C. (1984). The formation of ability conceptions: developmental trend or social construction? Review of Educational Research, 54, 31-63.

Stipek, D. C. \& Daniels, D.H. (1988). Declining perceptions of competence: a consequence of changes in the child or in the educational environment? Journal of Educational Psychology, 80, 352-356.

Taliuli, N. (1982). Atribuição de causalidade em tarefas acadêmicas por alunos de nivel sócio-econômico baixo e desempenho diferente. Dissertação de Mestrado nãopublicada. Curso de Psicologia, Universidade Federal do Espírito Santo. Vitória, Espírito Santo.

Weiner, B. (1979). A theory of motivation for some classroom experiences. Journal of Educational Psychology, 71, 3-25.

Weiner, B. (1985). An attributional theory of achievement motivation and emotion. Psychological Review, 92, 548-573.

Weiner, B. (1993). On sin versus sickness: A theory of perceived responsibility and social motivation. American Psychologist, 48, 957-965.

Recebido: 08/11/2000

Revisado: 06/03/2001

Aceite Final: 08/05/2001

Sobre a autora

Evely Boruchovitch é Psicóloga pela Universidade do Estado do Rio de Janeiro, Ph.D. em Educação pela University of Southern California, Professora do Departamento de Psicologia Educacional da Faculdade de Educação da Universidade Estadual de Campinas e do Programa de Pós-Graduação em Educação da Universidade São Francisco. 